\title{
Spontaneous adipogenic differentiation potential of adipose-derived stem cells decreased with increasing cell passages
}

\author{
DUO YANG, NA LI and GUOYING ZHANG
}

\author{
Central Laboratory, Beijing Shijitan Hospital of Capital Medical University, Beijing 100038, P.R. China
}

Received October 10, 2017; Accepted February 9, 2018

DOI: $10.3892 / \mathrm{mmr} .2018 .8619$

\begin{abstract}
Primary adipose-derived stem cells (ADSCs) are a mixture of cell types including preadipocytes having the ability to spontaneously differentiate into adipocytes. The aim of the present study was to compare the spontaneous adipogenic differentiation potential of ADSCs at different passages to determine whether it decreased with continuous cell passages. Mouse ADSCs (mADSCs) were harvested and cells from passages 1 to 5 were used for experiments. The proliferation of mADSCs at different passages was tested using the cell counting kit- 8 assay. Reverse transcription-quantitative polymerase chain reaction was used to determine relative mRNA expression levels of CCAAT/enhancer binding protein $\alpha$ (Cebpa and $\mathrm{C} / \mathrm{EBP} \alpha$ ) and peroxisome proliferator-activated receptor $\gamma$ (Pparg and PPAR $\gamma$ ), and western blot analysis was used to investigate $\mathrm{C} / \mathrm{EBP} \alpha$ and $\operatorname{PPAR} \gamma$ protein expression. The cells were cultured using DMEM. The fixed cells were then stained using Oil Red $\mathrm{O}$ on days 14 and 28, and the obtained extracted dye was monitored for absorbance. The $510 \mathrm{~nm}$ absorbance from passages 1 to 5 was observed to be statistically different. The relative expression levels of Cebpa and Pparg for mADSCs from passage 1 were significantly higher when compared with those for mADSCs from passages 2 to 5 on days 3,5 and 7. However, no difference was identified in the expression levels of proteins $\mathrm{C} / \mathrm{EBP} \alpha$ and PPAR $\gamma$ for different passages. Although the mRNA expression levels of Pparg from passages 4 to 5 were significantly higher when compared with those from passage 1, the results of Oil Red $\mathrm{O}$ absorbance, mRNA expression levels of Cebpa, and the protein expression levels of $\mathrm{C} / \mathrm{EBP} \alpha$ and PPAR $\gamma$ exhibited no difference between mADSCs from passages 1 to 5 when cultured with induced adipogenic differentiation medium. Therefore, it was concluded that the spontaneous adipogenic
\end{abstract}

Correspondence to: Dr Duo Yang, Central Laboratory, Beijing Shijitan Hospital of Capital Medical University, 10 Yangfangdian Tieyi, Haidian, Beijing 100038, P.R. China

E-mail: yangduo@bjmu.edu.cn

Key words: adipogenic differentiation, adipose-derived stem cells, proliferation, spontaneous adipogenic differentiation differentiation potential of ADSCs decreased with continuous cell passages.

\section{Introduction}

Adipose-derived stem cells (ADSCs) can differentiate into a variety of cell types, including adipocytes, osteoblasts, chondrocytes and myocytes $(1,2)$. They are widely used in regenerative medicine (3-7). The primary ADSCs from adipose tissue are a mixture of cell types, including preadipocytes, having the ability to spontaneously differentiate into adipocytes $(1,2)$. The spontaneous potential of preadipocytes to differentiate into adipocytes may compromise the proliferation and/or differentiation potential of $\operatorname{ADSCs}(8,9)$, especially in chondrogenic or osteogenic differentiation. The influence of spontaneous adipogenic differentiation should be minimized in chondrogenic or osteogenic regeneration, but it does not seem to be harmful in adipogenic regenerative medicine. Thus, understanding how the potential of spontaneous adipogenic differentiation of ADSCs changes may be worthy of tissue regeneration.

The heterogeneity of cultured ADSCs is reduced quickly by continuous cell passages $(8,10-12)$. Whether the spontaneous adipogenic differentiation is affected by increasing number of cell passages is not clear so far. The spontaneous adipogenic differentiation of ADSCs was assumed to decrease when the cells were passaged serially. ADSCs from passages 3 and 4 were reported to be used in regenerative medicine (13-15). Krähenbühl et al (16) reported that cell morphology changed and growth slowed down when extended to passages 6 and beyond. Therefore, ADSCs from passages 1 to 5 was explored in this study to observe the change in spontaneous adipogenic differentiation for cells from different passages.

The induced adipogenic potential of ADSCs was reported to decrease with increasing passage number (17). Therefore, the induced adipogenic differentiation potentials at different passages were also studied and compared in the present study.

Peroxisome proliferator-activated receptor $\gamma(\operatorname{PPAR} \gamma)$ and CCAAT/enhancer-binding protein $\alpha(\mathrm{C} / \mathrm{EBP} \alpha)$ have been demonstrated to be vital in adipocyte differentiation and these two transcription factors cooperate on multiple binding sites in promoter regions and regulate a wide range of genes expressed in developing and mature adipocytes (18). This study observed Cebpa and Pparg as adipocyte marker genes in evaluating the spontaneous and induced adipogenic differentiation of ADSCs 
and explored its potential to evaluate the feasibility of ADSCs from passages 1 to 5 and facilitate the selection of optimal cell passage for regenerating different tissues.

\section{Materials and methods}

Chemicals, antibodies and dyes. 3-Isobutyl-1-methylxanthine (IBMX), dexamethasone, indomethacin, insulin and Oil Red O were purchased from Sigma-Aldrich (Merck KGaA, Darmstadt, Germany). Rabbit anti-Ppar $\gamma$ and anti-CEBP $\alpha$ antibodies were procured from Abcam (Cambridge, MA, USA).

Isolation and culture of mouse ADSCs. All C57BL/6 mice aged 7-10 days, purchased from Weitong-Lihua Laboratory (Beijing, China), were age- and sex-matched for each experiment. All animal experiments were approved by the Institutional Animal Care and Use Committee of Beijing Shijitan Hospital of Capital Medical University, Beijing, China (no. 2017-KL16).

Mouse ADSCs (mADSCs) were isolated and cultured as described in a previous study (19). In brief, the subcutaneous adipose tissue of C57BL/6 mice was cut into small pieces and digested with collagenase I in phosphate-buffered saline (PBS) for $1 \mathrm{~h}$ at $37^{\circ} \mathrm{C}$. The cells were then washed and filtered through a $70-\mu \mathrm{m}$ cell strainer, resulting in a single-cell suspension. Isolated cells were then seeded at a density of $3-4 \times 10^{4}$ cells $/ \mathrm{cm}^{2}$ in a culture vessel containing low-glucose Dulbecco's modified Eagle's medium (DMEM) supplemented with 10\% fetal bovine serum (FBS) (both from Gibco; Thermo Fisher Scientific, Inc., Waltham, MA, USA) and 1\% antibiotic-antimycotic solution (Invitrogen; Thermo Fisher Scientific, Inc.), which favored the attachment and growth of fibroblastic cells (20). The attached cells were washed and replenished using a fresh medium every $48 \mathrm{~h}$ to discard unattached dead cells or immune cells, and by days $3-5,>90 \%$ cells displayed typical fibroblastic morphology. These adherent cells were named primary ADSCs (P0). Cell passaging was performed using a $0.25 \%$ trypsin solution (Sigma-Aldrich) every 3-5 days when the attached cells reached $80-90 \%$ confluence and cells were seeded at $3 \times 10^{4}$ cells/ $/ \mathrm{cm}^{2}$ per passage for a total of 5 passages.

For induced adipogenic differentiation, one-day postconfluence cells were switched to adipogenic differentiation medium [DMEM with FBS (10\%), $0.5 \mathrm{mM}$ IBMX, $1 \mu \mathrm{M}$ dexamethasone, $10 \mu \mathrm{M}$ insulin, $200 \mu \mathrm{M}$ indomethacin, and $1 \%$ antibiotic/antimycotic] to induce differentiation as described in a previous study (21). The cells were collected at indicated time points for RNA and protein extraction.

Oil Red $O$ staining. The cells were harvested on the induction day according to the experimental design. They were rinsed with PBS twice and fixed with $4 \%$ polyoxymethylene in PBS (w/v) for $30 \mathrm{~min}$ at room temperature. Oil Red O $(0.5 \mathrm{~g}$; Amresco, LLC, Solon, OH, USA) was dissolved in isopropanol $(100 \mathrm{ml}, \mathrm{w} / \mathrm{v})$, diluted with water $(6: 4, \mathrm{v} / \mathrm{v})$, and filtered. The fixed cells were then stained with the filtered Oil Red O solution for $1 \mathrm{~h}$ at room temperature and exhaustively rinsed with water. Excess water was evaporated by placing the stained cultures at a temperature of $25-30^{\circ} \mathrm{C}$. Then, $0.8 \mathrm{ml}$ of isopropyl alcohol was added to the stained culture dish. The extracted dye was immediately removed by gentle pipetting, and its absorbance monitored spectrophotometrically at $510 \mathrm{~nm}$ as described in a previous study (22).

Reverse transcription-quantitative polymerase chain reaction $(R T-q P C R)$. Total cellular RNA was extracted using the RNAeasy reagent (Qiagen GmbH, Hilden, Germany), and $500 \mathrm{ng}$ of RNA was reverse transcribed to cDNA using a cDNA synthesis kit (ComWin, Beijing, China). qPCR was performed using the QuantStudio 6 Flex 96 real-time system (Applied Biosystems; Thermo Fisher Scientific, Inc.) using SYBR-Green Mix (ComWin). All the primers used with SYBR-Green were designed to span at least one exon to minimize the possibility of nonspecific amplification from the genomic DNA. Glyceraldehyde-3-phosphate dehydrogenase (GAPDH) gene was used as a housekeeping gene to normalize data. Specific primer sequences were used as described in a previous study (19) and are shown in Table I.

Western blot analysis. The total protein was extracted from harvested cells using radioimmune precipitation assay buffer supplemented with $1 \%$ phenylmethanesulfonyl fluoride (Applygen, Beijing, China), and the concentration was quantified using a Bicinchoninic Acid protein assay kit (Thermo Fisher Scientific Inc.). Then, $40 \mu \mathrm{g}$ of the extracted protein was subjected to sodium dodecyl sulfate-polyacrylamide gel electrophoresis (Applygen), transferred onto a nitrocellulose membrane, blocked with $0.5 \%$ skimmed milk overnight, washed with Tris-buffered saline with Tween-20 buffer, and incubated with primary antibodies (anti-C/EBP $\alpha$, anti-PPAR $\gamma$ and anti-GAPDH at a dilution of 1:1,000; Abcam, Cambridge, UK) for $18 \mathrm{~h}$ at $4^{\circ} \mathrm{C}$. Then, the membranes were washed and incubated with 1:10,000 diluted goat anti-rabbit immunoglobulin G H\&L conjugated with Alexa Fluor (Abcam). The signals were detected using Odyssey CLx (LI-COR Bioscience, Lincoln, NE, USA). Western blot data were quantified using the ImageJ version 1.38 software (HYPERLINK https://imagej.nih.gov/ij/). The ratio of target protein/GAPDH indicated the relative expression of target protein.

Cell counting kit-8 assay (CCK-8). The proliferation of $\mathrm{mADSCs}$ at different passages in vitro was tested via measuring the number of living cells using the CCK-8 (Dojindo, Tokyo, Japan) according to the manufacturer's instruction. Briefly, mADSCs were seeded in 96-well plates (2,000 cells/well). After $2 \mathrm{~h}$ (named day 0 in the CCK-8 assay in this study), $24,48,72,96$ and $120 \mathrm{~h}$, the cells were incubated with the CCK8 solution for $4 \mathrm{~h}$ at $37^{\circ} \mathrm{C}$ until the optical density values at $450 \mathrm{~nm}(\mathrm{OD} 450)$ were read using a microplate reader. The experiments were repeated three times.

Statistical analysis. Experiments were repeated at least three times with similar results. Graph data represented the mean \pm standard error calculated from independent experiments. Statistical significance was performed with SPSS 20.0 (IBM Corp., Armonk, NY, USA) using the one-way analysis of variance (ANOVA) followed by the Bonferroni post hoc test, if it was necessary. The results were analyzed and graphs built using GraphPad Prism version 5.02 (GraphPad Software, Inc., La Jolla, CA, USA). 
Table I. List of primers used for reverse transcription-quantitative polymerase chain reaction.

\begin{tabular}{lll}
\hline Gene & Strand & \multicolumn{1}{c}{ Primer sequence (5'-3') } \\
\hline Cebpa & Forward & CAAGAACAGCAACGAGTACCG \\
& Reverse & GTCACTGGTCAACTCCAGCAC \\
Ppary & Forward & TCGCTGATGCACTGCCTATG \\
& Reverse & GAGAGGTCCACAGAGCTGATT \\
GAPDH & Forward & AACTTTGGCATTGTGGAAGG \\
& Reverse & ACACATTGGGGGTAGGAACA \\
\hline
\end{tabular}

Cebpa, CCAAT/enhancer binding protein $\alpha$; Ppar $\gamma$, peroxisome proliferator-activated receptor $\gamma$.

\section{Results}

Proliferation of $m A D S C s$ at different passages in vitro. It was reported that ADSCs became senescent, and their proliferation and differentiation potentials reduced after the cells were passaged multiple times $(23,24)$. Therefore, the proliferation potential of cells from passages 1 to 5 was investigated. The proliferation of mADSCs at different passages in vitro was evaluated using the CCK-8 assay. Fig. 1 represents the results. The proliferation curves of mADSCs at different passages were similar from day 0 to 5 . Also, no difference was found in the $450-\mathrm{nm}$ absorbance on days 0 to 5 between mADSCs at different passages. The results suggested that several cell passages had no significant effect on the proliferation of mADSCs.

Spontaneous adipogenic differentiation potential of mADSCs at different passages in vitro. Preadipocytes in the primary ADSCs tended to undergo adipogenic differentiation spontaneously $(1,2)$. Also, the heterogeneity of ADSCs reduced with cell passages (11). The spontaneous adipogenic differentiation potential of mADSCs at different passages was observed to find out whether it decreased with increasing cell passages.

Spontaneous adipogenic differentiation potential of mADSCs at different passages in vitro was examined by staining with Oil Red O, and the relative expression levels of Cebpa and Pparg were determined using real-time PCR and western blot analysis. The mADSCs at different passages were seeded at a density of $3 \times 10^{4}$ cells $/ \mathrm{cm}^{2}$ in a culture vessel containing DMEM with $10 \%$ serum and antibiotics. The timing of the experiment was recorded as day 0 one-day after confluence. Then, the cells were washed and replenished using a fresh medium every $48 \mathrm{~h}$. Without treatment with adipogenic differentiation medium and without further passaging, the cells were collected on days $0,3,5$ and 7 for RNA extraction and collected on day 7 for protein extraction. The cells were stained with Oil Red O on days 14 and 28 (Fig. 2A), and the absorbance was monitored at $510 \mathrm{~nm}$. Without induced adipogenic differentiation, the spontaneous adipogenic potential of mADSCs at different passages was different in vitro. The absorbance of extracted dye after staining with Oil Red O on days 14 and 28 was statistically different (Fig. 2B). The 510-nm absorbance obtained for mADSCs from passage 1 was higher than that

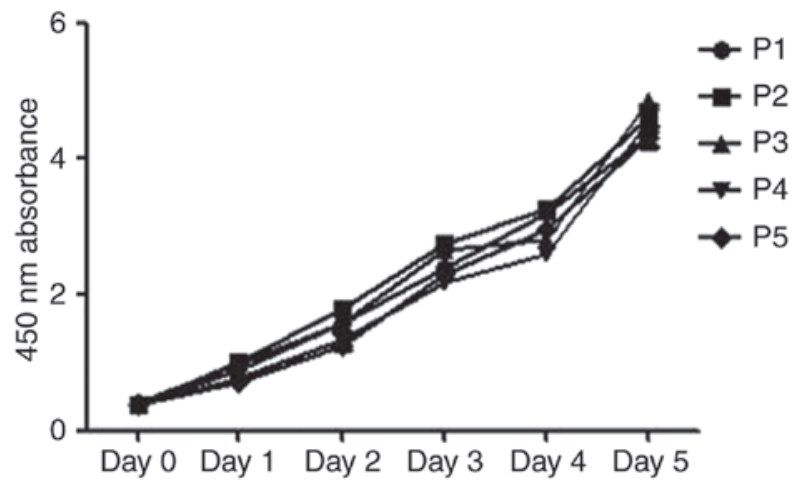

Figure 1. Proliferation of mADSCs at different passages in vitro. The proliferation of mADSCs at different passages in vitro was evaluated using the cell counting kit- 8 assay. The proliferation curves were similar from days 0 to 5 . No difference in $450 \mathrm{~nm}$ absorbance was observed between mADSCs at different passages. Data were obtained from independent triplicate experiments, and are presented as the mean \pm standard error mean. P, passage; mADSCs, mouse adipose-derived stem cells.

for mADSCs from passages 2 to 5 . However, no difference in absorbance was found between mADSCs from passages 2 to 5 on days 14 and 28. The relative expression levels of Cebpa and Pparg from different passages were undifferentiated on day 0 , but the expression levels of these both genes from passage 1 were significantly higher compared with those of mADSCs from passages 2 to 5 on days 3, 5 and 7 (Fig. 2C). However, no difference in the expression levels of C/EBP $\alpha$ and PPAR $\gamma$ proteins was observed between mADSCs from passages 1 to 5 on day 7 (Fig. 2D).

Therefore, mADSCs had the potential to undergo spontaneous adipogenic differentiation, and the adipogenic differentiation potential decreased quickly with several cell passages. Then, the induced adipogenic differentiation potential of mADSCs at different passages (cells cultured with adipogenic differentiation medium) was compared to know whether this induced differentiation was also affected by the number of cell passages.

Induced adipogenic differentiation of mADSCs at different passages in vitro. The mADSCs were cultured with adipogenic differentiation medium to detect whether the adipogenesis of mADSCs at different passages under induced adipogenic differentiation was discrepant. The timing of the experiment was recorded as day 0 one day after the confluence, then cells were switched to adipogenic differentiation medium. The mRNA expression levels of Cebpa and Pparg were analyzed on days $0,3,5$ and 7 . The cells were collected for detecting the protein expression levels of C/EBP $\alpha$ and PPAR $\gamma$ on day 7, and the extracted dye was monitored after staining with Oil Red O on days 7 and 14. The real-time PCR analysis showed that the mRNA expression levels of Pparg for mADSCs from passages 3 to 5 were significantly higher than those for mADSCs from passage 1 on days 3, 5 and 7 . However, no difference in the expression level of Cebpa was observed between mADSCs at different passages on days 3,5 and 7 (Fig. 3C). The results of Oil Red O absorbance showed no difference between cells from passages 1 to 5 on days 7 and 14 under induced adipogenic differentiation (Fig. 3A and B). Also, western blot analysis showed no difference in the protein 
A

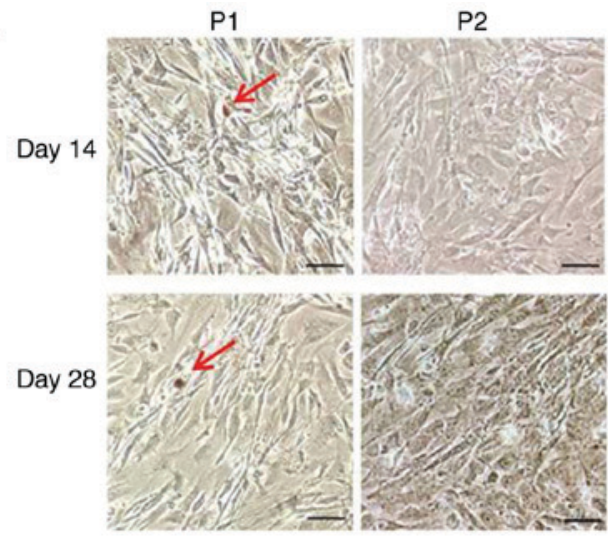

P3

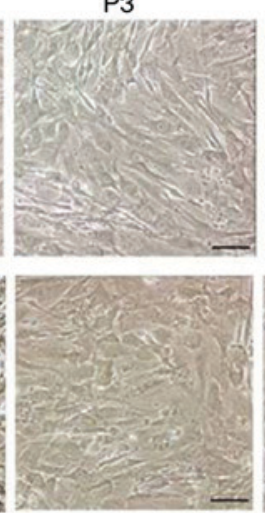

P4

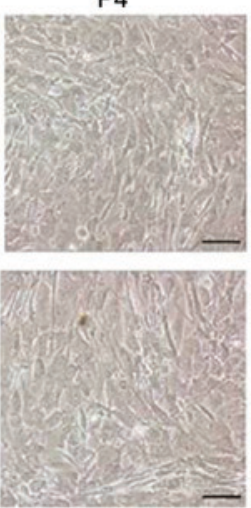

P5

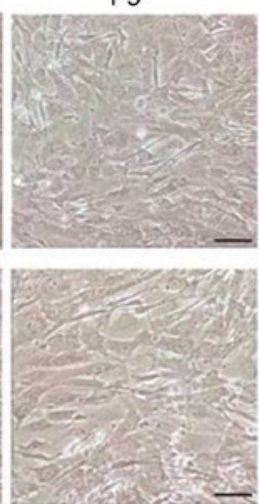

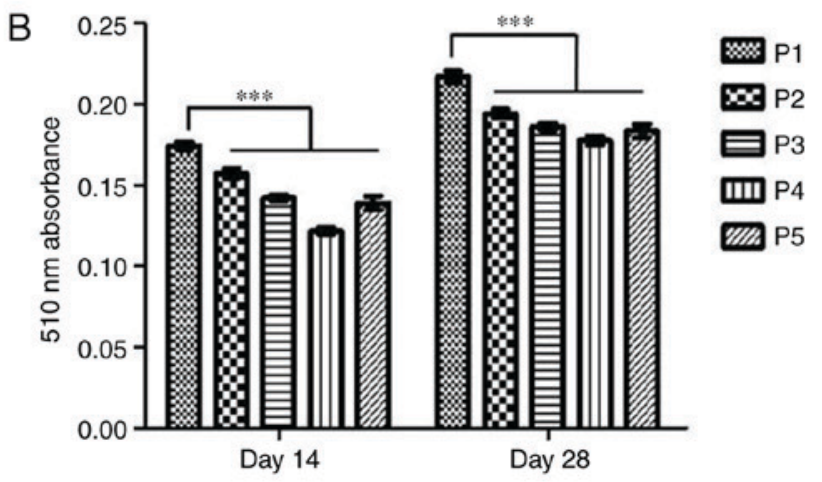

C
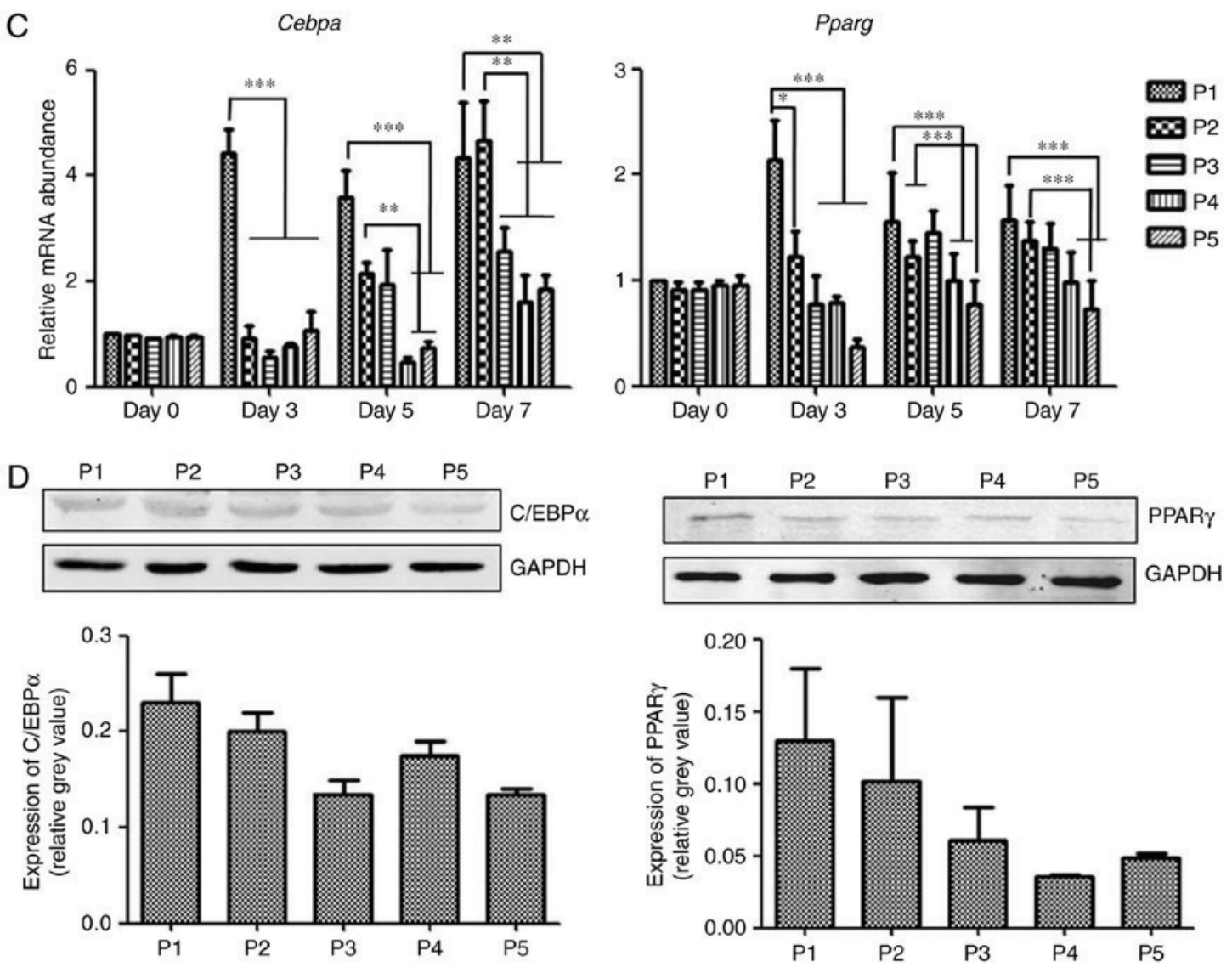

Figure 2. Spontaneous adipogenic differentiation potential of mADSCs at different passages in vitro. (A) Oil Red O staining of mADSCs on days 14 and 28 without induced adipogenic differentiation. Scale bars, $100 \mu \mathrm{m}$; magnification, $\mathrm{x} 100$. The arrows indicate the lipids present in adipocytes (red staining). (B) The absorbance of extracted dye from mADSCs at different passages following staining with Oil Red O on days 14 and 28 . (C) Without adipogenic differentiation, the relative expression levels of Cebpa and Ppar $\gamma$ from mADSCs at different passages were detected on days 0, 3, 5 and 7. Expression levels of these 2 genes from P1 were significantly higher when compared with those from P2 to 5 on days 3,5 and 7. (D) Expression of C/EBP $\alpha$ and PPAR $\gamma$ proteins was detected on day 7 using western blot analysis. No difference was identified in the protein expression levels of mADSCs at different passages. Experiments were repeated at least 3 times with similar results. Data are presented as the mean \pm standard error of mean. ${ }^{*} \mathrm{P}<0.05,{ }^{* *} \mathrm{P}<0.01$ and ${ }^{* * * *} \mathrm{P}<0.001$, as indicated. $\mathrm{P}$, passage; $\mathrm{mADSCs}$, mouse adipose-derived stem cells; Cebpa and C/EBP $\alpha$, CCAAT/enhancer binding protein $\alpha$; PPAR $\gamma$ and Pparg, peroxisome proliferator-activated receptor $\gamma$. 

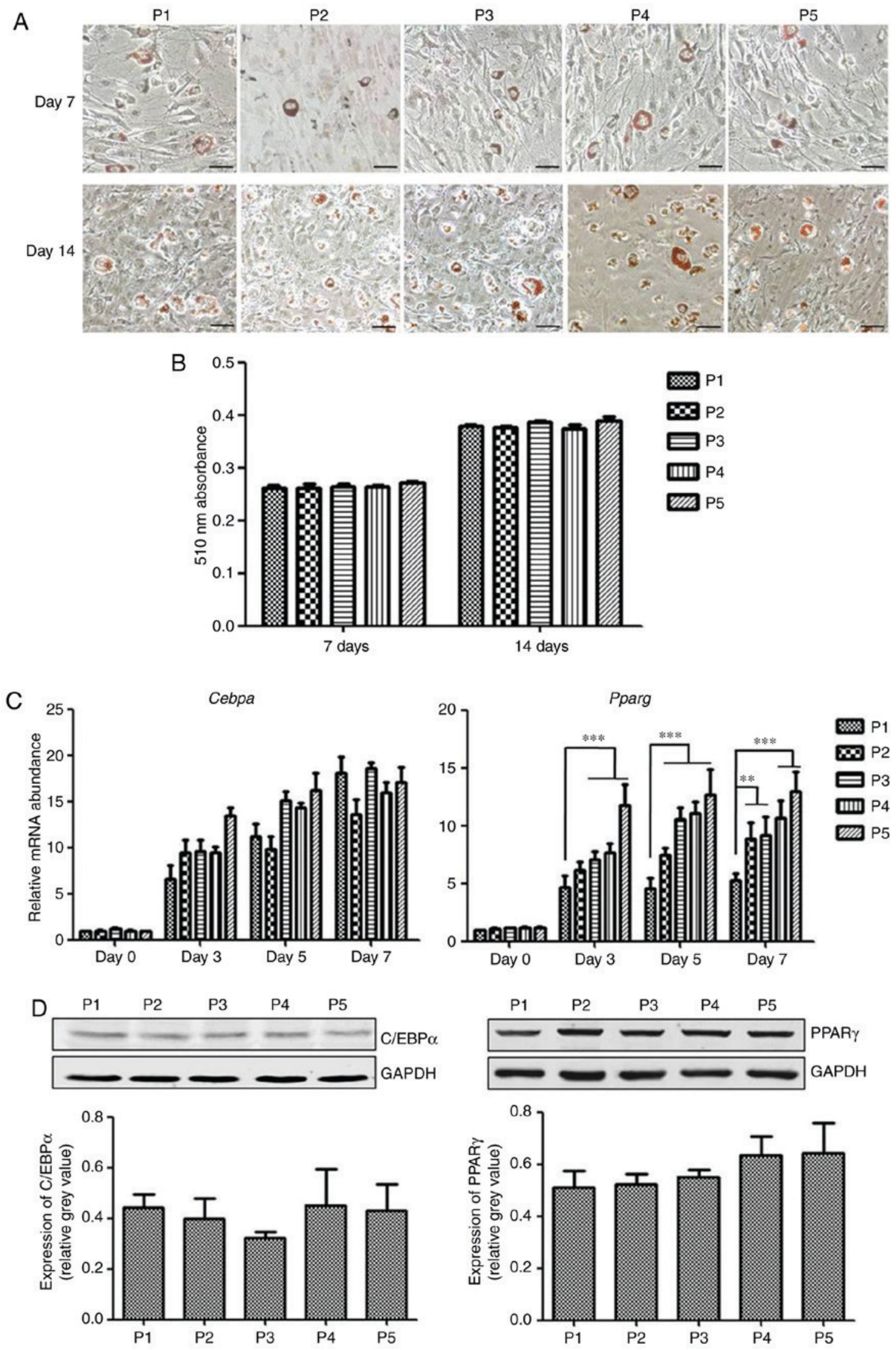

Figure 3. Adipogenic differentiation of mADSCs at different passages in vitro. (A) mADSCs cultured with adipogenic differentiation medium on days 7 and 14 . Lipid production was shown using Oil Red O staining. Scale bars, $100 \mu \mathrm{m}$; magnification, x100. (B) Absorbance of extracted dye following mADSCs were stained with Oil Red O; the absorbance of extracted dye from mADSCs at different passages was undifferentiated on days 7 and 14 . (C) Cultured with adipogenic differentiation medium, the relative expression levels of Cebpa and Pparg from mADSCs at different passages were detected on days $0,3,5$ and 7. (D) Western blot analysis revealed the expression levels of C/EBP $a$ and PPAR $\gamma$ proteins under induced adipogenic differentiation on day 7. Experiments were repeated at least three times with similar results. Data are presented as the mean \pm standard error of mean. ${ }^{* *} \mathrm{P}<0.01$ and ${ }^{* * *} \mathrm{P}<0.001$, as indicated. $\mathrm{P}$, passage; mADSCs, mouse adipose-derived stem cells; Cebpa and C/EBP $\alpha$, CCAAT/enhancer binding protein $\alpha$; PPAR $\gamma$ and Pparg, peroxisome proliferator-activated receptor $\gamma$. 
expression levels of $\mathrm{C} / \mathrm{EBP} \alpha$ and PPAR $\gamma$ between mADSCs at different passages on day 7 (Fig. 3D).

Hence, the induced adipogenic differentiation potential was not significantly different between mADSCs from passages 1 to 5 (except the expression levels of Ppary). Thus, the induced adipogenic differentiation was not affected significantly by limited continuous cell passages.

\section{Discussion}

Generally, ADSCs are isolated in pools that contain a mixture of cell types including mesenchymal stem cells and other types of cells such as preadipocytes (2). Preadipocytes, which are the specific precursor cells, have the potential for spontaneous adipogenic differentiation. For more effective use of ADSCs, how the adipogenic differentiation of ADSCs is influenced by preadipocytes needs to be understood and the influence of spontaneous adipogenic differentiation needs to be minimized when ADSCs are used in regenerative medicine.

The content of preadipocytes is less in the primary ADSCs 'mix' $(23,25,26)$ and preadipocytes share several cell markers with the primary adipose derived stem cells, like Lin ${ }^{-}, \mathrm{CD}_{2} 9^{+}$, CD $34^{+}, \mathrm{Sca}^{-} 1^{+}$, and CD45- ${ }^{-} \mathrm{CD} 31^{-}(23,25)$, making it difficult to screen for preadipocytes alone from the primary ADSC 'mix' using fluorescence-activated cell sorting. In the clinical practice of regenerative medicine, generally, the ADSCs was used and observed as a whole (3-6). For these reasons above, in this study, the primary ADSC 'mix' was observed as a whole instead of screening for preadipocytes. The potential of spontaneous adipogenic differentiation of ADSC 'mix' was investigated in this study.

Culturing with increasing cell passages was found to reduce the heterogeneity of ADSCs (11). Studies indicated that continuous cell passages reduced the number of cells with a CD34 ${ }^{+}$surface marker, which differentiated into adipocytes more efficiently $(25,27)$. However, whether spontaneous adipogenic differentiation of ADSCs would decrease with serial cell passages is not yet clear.

In this study, the spontaneous and induced adipogenic differentiation potentials of mADSCs at different passages were explored in vitro. ADSCs were found to differentiate into adipocytes spontaneously, and the ability of spontaneous differentiation decreased quickly with the increase in the number of cell passages.

The ability of spontaneous adipogenic differentiation can have clinical implications, which may be beneficial or damaging $(8,28)$. For chondrogenic or osteogenic regeneration, the spontaneous adipogenic differentiation of ADSCs should be minimized as possible. Adipogenic, chondrogenic, and osteogenic differentiation was observed in passage 3 in many studies (29-34). The results of the present study indicated that the impact of spontaneous adipogenic differentiation reduced sufficiently after passage 3 , and the cells passaged more than three times were probably suitable for chondrogenic and osteogenic regeneration. For adipogenic regeneration, the early passages of ADSCs may be more suitable.

The adipogenic differentiation potential was correlated with the number of mesenchymal stem cells in the ADSC 'mix'. No significant difference was found between the adipogenic differentiation potential of cells from passages 1 to 5 , suggesting that the adipogenic differentiation of mesenchymal stem cells was not affected by continuous cell passages. Liu et al (17) reported that the adipogenic potential of ADSCs decreased and the osteogenic differentiation increased when the cells were passaged more than 12 times. In this study, the adipogenic differentiation potential was not significantly different between cells from passages 1 to 5 (except the expression levels of Pparg) suggesting that the adipogenic potential of ADSCs was not affected by limited cell passages.

ADSCs from passages 3 and 4 were reported to be used in regenerative medicine (13-15). The results of this study also indicated no significant difference in spontaneous and induced adipogenic differentiation potential between cells of passages 3 and 4. It suggested similar differentiation results in adipogenic regeneration when using cells from passages 3 and 4 . Whether the potential of chondrogenic and osteogenic differentiation from cells of passages 3 and 4 is different needs a follow-up study.

This study also analyzed the proliferation of mADSCs at different passages. No difference in proliferation was observed between cells from passages 1 to 5 . Kim et al (35) reported that long-term culture and cell passages of ADSCs could impair the activity of cells and provoke cellular senescence, leading to low efficacy in vivo. The results indicated that several cell passages had no significant effect on the proliferation of mADSCs.

In conclusion, the present study showed that mADSCs could undergo adipogenic differentiation spontaneously, and the differentiation potential decreased with continuous cell passages. The induced adipogenic differentiation was not affected significantly by cell passages. The proliferation was not affected by limited cell passages.

\section{Acknowledgements}

Not applicable.

\section{Funding}

No funding was received.

\section{Availability of data and materials}

The datasets used and analysed during the present study are available from the corresponding author on reasonable request.

\section{Authors' contributions}

NL isolated and cultured mouse adipose-derived stem cells. GZ performed the oil red staining. NL and GZ statistically analyzed and interpreted the data. DY designed the study, performed the reverse transcription-quantitative polymerase chain reaction, western blotting and statistical analysis, and was a major contributor in writing the manuscript. All authors read and approved the final manuscript.

\section{Ethics approval and consent to participate}

All animal experiments were approved by the Institutional Animal Care and Use Committee of Beijing Shijitan Hospital of Capital Medical University (Beijing, China) (approval no. 2017-KL16). 


\section{Consent for publication}

Not applicable.

\section{Competing interests}

The authors declare that they have no competing interests.

\section{References}

1. Rodeheffer MS, Birsoy K and Friedman JM: Identification of white adipocyte progenitor cells in vivo. Cell 135: 240-249, 2008.

2. Yoshimura K, Shigeura T, Matsumoto D, Sato T, Takaki Y, Aiba-Kojima E, Sato K, Inoue K, Nagase T, Koshima I, et al: Characterization of freshly isolated and cultured cells derived from the fatty and fluid portions of liposuction aspirates. J Cell Physiol 208: 64-76, 2006.

3. Bunnell BA, Flaat M, Gagliardi C, Patel B and Ripoll C: Adipose-derived stem cells: Isolation, expansion and differentiation. Methods 45: 115-120, 2008

4. Gimble JM, Guilak F and Bunnell BA: Clinical and preclinical translation of cell-based therapies using adipose tissue-derived cells. Stem Cell Res Ther 1: 19, 2010.

5. Gir P, Oni G, Brown SA, Mojallal A and Rohrich RJ: Human adipose stem cells: Current clinical applications. Plast Reconstr Surg 129: 1277-1290, 2012.

6. Harasymiak-Krzyżanowska I, Niedojadło A, Karwat J, Kotula L, Gil-Kulik P, Sawiuk M and Kocki J: Adipose tissue-derived stem cells show considerable promise for regenerative medicine applications. Cell Mol Biol Lett 18: 479-493, 2013.

7. Tobita M, Orbay $\mathrm{H}$ and Mizuno $\mathrm{H}$ : Adipose-derived stem cells: Current findings and future perspectives. Discov Med 11: 160-170, 2011

8. Romagnoli C and Brandi ML: Adipose mesenchymal stem cells in the field of bone tissue engineering. World J Stem Cells 6 : 144-152, 2014

9. Roxburgh J, Metcalfe AD and Martin YH: The effect of medium selection on adipose-derived stem cell expansion and differentiation: Implications for application in regenerative medicine. Cytotechnology 68: 957-967, 2016.

10. Baer PC and Geiger H: Adipose-derived mesenchymal stromal/stem cells: Tissue localization, characterization, and heterogeneity. Stem Cells Int 2012: 812693, 2012.

11. Locke M, Windsor J and Dunbar PR: Human adipose-derived stem cells: Isolation, characterization and applications in surgery. ANZ J Surg 79: 235-244, 2009.

12. Fu Y, Deng J, Jiang Q, Wang Y, Zhang Y, Yao Y, Cheng F, Chen X, Xu F, Huang M, et al: Rapid generation of functiona hepatocyte-like cells from human adipose-derived stem cells. Stem Cell Res Ther 7: 105, 2016

13. Orbay H, Devi K, Williams PA, Dehghani T, Silva EA and Sahar DE: Comparison of endothelial differentiation capacities of human and rat adipose-derived stem cells. Plast Reconstr Surg 138: 1231-1241, 2016.

14. Dufrane D and Lafosse A: A simple method to determine the purity of adipose-derived stem cell-based cell therapies. Stem Cells Transl Med 5: 1575-1579, 2016.

15. Lin YC, Harn HJ, Lin PC, Chuang MH, Chen CH, Lin SZ and Chiou TW: Commercial production of autologous stem cells and their therapeutic potential for liver cirrhosis. Cell Transplant 26 : 449-460, 2017

16. Krähenbühl SM, Grognuz A, Michetti M, Raffoul W and Applegate LA: Enhancement of human adipose-derived stem cell expansion and stability for clinical use. Int J Stem Cell Res Ther 2: 7, 2015.

17. Liu Y, Zhang Z, Zhang C, Deng W, Lv Q, Chen X, Huang T and Pan L: Adipose-derived stem cells undergo spontaneous osteogenic differentiation in vitro when passaged serially or seeded at low density. Biotech Histochem 91: 369-376, 2016.
18. Lowe CE, O'Rahilly S and Rochford JJ: Adipogenesis at a glance. J Cell Sci 124: 2681-2686, 2011.

19. Zhang LJ, Guerrero-Juarez CF, Hata T, Bapat SP, Ramos R, Plikus MV and Gallo RL: Innate immunity. Dermal adipocytes protect against invasive Staphylococcus aureus skin infection. Science 347: 67-71, 2015

20. Muto J, Morioka Y, Yamasaki K, Kim M, Garcia A, Carlin AF, Varki A and Gallo RL: Hyaluronan digestion controls DC migration from the skin. J Clin Invest 124: 1309-1319, 2014

21. Zuk PA, Zhu M, Ashjian P, De Ugarte DA, Huang JI, Mizuno H, Alfonso ZC, Fraser JK, Benhaim P and Hedrick MH: Human adipose tissue is a source of multipotent stem cells. Mol Biol Cell 13: 4279-4295, 2002

22. Ramirez-Zacarías JL, Castro-Muñozledo F and Kuri-Harcuch W: Quantitation of adipose conversion and triglycerides by staining intracytoplasmic lipids with Oil Red O. Histochemistry 97: 493-497, 1992.

23. Bajek A, Gurtowska N, Olkowska J, Kazmierski L, Maj M and Drewa T: Adipose-derived stem cells as a tool in cell-based therapies. Arch Immunol Ther Exp (Warsz) 64: 443-454, 2016.

24. de Witte SF, Lambert EE, Merino A, Strini T, Douben HJ, O'Flynn L, Elliman SJ, de Klein AJ, Newsome PN, Baan CC, et al: Aging of bone marrow- and umbilical cord-derived mesenchymal stromal cells during expansion. Cytotherapy 19: 798-807, 2017.

25. Bajek A, Gurtowska N, Olkowska J, Maj M, Kaźmierski Ł, Bodnar M, Marszałek A, Debski R and Drewa T: Does the harvesting technique affect the properties of adipose-derived stem cells?-the comparative biological characterization. J Cell Biochem 118: 1097-1107, 2017.

26. González-Cruz RD and Darling EM: Adipose-derived stem cell fate is predicted by cellular mechanical properties. Adipocyte 2: 87-91, 2013.

27. Wang QF, Huang Y, He GC, Wang HS, Chen ZH, Cai XH, Xie YH and Liu Q: Osteoblast differentiation of rabbit adipose-derived stem cells by polyethylenimine-mediated BMP-2 gene transfection in vitro. Genet Mol Res 16, 2017.

28. Aoyagi Y, Kuroda M, Asada S, Bujo H, Tanaka S, Konno S, Tanio M, Ishii I, Aso M and Saito Y: Fibrin glue increases the cell survival and the transduced gene product secretion of the ceiling culture-derived adipocytes transplanted in mice. Exp Mol Med 43: 161-167, 2011.

29. Güven S, Mehrkens A, Saxer F, Schaefer DJ, Martinetti R, Martin I and Scherberich A: Engineering of large osteogenic grafts with rapid engraftment capacity using mesenchymal and endothelial progenitors from human adipose tissue. Biomaterials 32: 5801-5809, 2011.

30. Jang Y, Jung H, Nam Y, Rim YA, Kim J, Jeong SH and Ju JH: Centrifugal gravity-induced BMP4 induces chondrogenic differentiation of adipose-derived stem cells via SOX9 upregulation. Stem Cell Res Ther 7: 184, 2016.

31. Papadimitropoulos A, Scherberich A, Güven S, Theilgaard N, Crooijmans HJ, Santini F, Scheffler K, Zallone A and Martin I: A 3D in vitro bone organ model using human progenitor cells. Eur Cell Mater 21: 445-458, 2011.

32. Leong DT, Abraham MC, Rath SN, Lim TC, Chew FT and Hutmacher DW: Investigating the effects of preinduction on human adipose-derived precursor cells in an athymic rat model. Differentiation 74: 519-529, 2006.

33. Maglione M, Spano S, Ruaro ME, Salvador E, Zanconati F, Tromba G and Turco G: In vivo evaluation of chitosan-glycerol gel scaffolds seeded with stem cells for full-thickness mandibular bone regeneration. J Oral Sci 59: 225-232, 2017.

34. Ye X, Liao C and Liu G: Age-related changes in the regenerative potential of adipose-derived stem cells isolated from the prominent fat pads in human lower eyelids 11: e0166590, 2016.

35. Kim S, Piao J, Son Y and Hong HS: Substance P enhances proliferation and paracrine potential of adipose-derived stem cells in vitro. Biochem Biophys Res Commun 485: 131-137, 2017. 